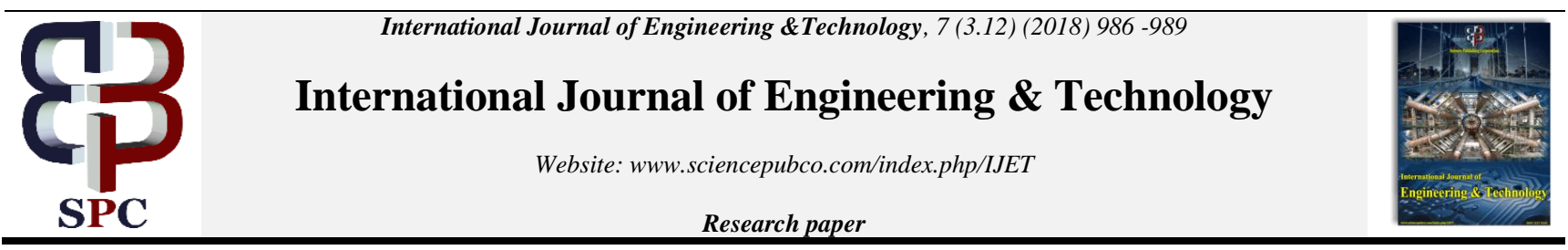

\title{
Transforming Cities from Simple to Smart using Internet of Things (IoT)
}

\author{
Anushka Khattri ${ }^{1}$, Alisha Khattri ${ }^{2}$, Ankita Kushwaha ${ }^{3}$, Rahul Chauhan ${ }^{4}$ \\ ${ }^{1,2,3}$ Computer Science and Engineering Department, Graphic Era Hill University, GEHU Dehradun, India \\ ${ }^{4}$ Electronics and Communication Engineering Department, Graphic Era Hill University, GEHU Dehradun, India \\ *Corresponding Author Email: ${ }^{1}$ anushkakhattri28@gmail.com , ${ }^{2}$ Khattriaarti2014@gmail.com \\ ${ }^{3}$ ankitakushwahaji@gmail.com, ${ }^{4}$ chauhan14853@gmail.com
}

\begin{abstract}
Dream of making a city smart is now transformed into reality through IoT. IoT is the best method to utilize the resources to the full extent. This paper deals with the basic but the very major problems such as navigation of parking lots. Every lock has a key and to avoid such problems intelligent sensors and cameras are used which can sense the input signals, then transforms it into data. These data are transmitted to cloud server which can provide the user about the real-time situation through mobile apps. For smart parking different methods such as expert system, wireless based system, GPS system and vehicular communication system are discussed in this paper.
\end{abstract}

Keywords: IoT, RFID, NB-IoT, GPS, WSN, smart city, sensors.

\section{Introduction}

The relationship between the technology and human is very strong, it is fair to say that the humans are completely reliable on machines but one should not forget that machines has no brain it does only what it was designed for, decisions are still made by the humans. To make right decision at right time well analyzation of the complete situation is needed which is wonderfully provided by the ICT (Information and Communication Technology) [4] and IoT (Internet of Things) for the urban development. The agenda for the upliftment of smart city is to improve the quality of living a life at affordable cost with the minimum consumption of resources. ICT and IoT [2] play a vital role in delivering the realtime information gathered by various sensors to the citizens. For better jobs and services, the migration rate from rural to urban areas is rapidly increasing which means more consumption of resource and more problems arise. To avoid the problems and also to sustain the resources for the future generation, effective use of ICT and IoT is required. This research paper specifically targets the problem faced in detection of parking lots which can be sorted not only with the support of smart technologies but also the contribution of the society equally plays a crucial role in this paradigm of smart city. For the implementation of smart parking expert system, wireless sensor-based system like magnetometer is used for the ground parking lot as it responds with the change of the earth's magnetic field due to vehicle, ultrasonic sensors [14] to detect vacant parking space in the multi-layered location, GPS system supports NAPA [16] to manage the status of the parking spaces and vehicle communication system [15] is used.

\section{A. 5 Key Elements of Smart City}

- Smart Energy: With the Renewable Source of energy, we can monitor and control the energy consumption.
- Smart Mobility: According to the Deloitte report that every year average American is stuck in traffic for about 34 hours. Smart Mobility means to have a smart solution to the problem.

- Smart Architecture: The infrastructure should be made to bear the dynamic problems and the existing system can be boost using the concept of IoT.

- Smart Public Services: Linking the residents of the city with the authorities by using the smart technologies make the city safer, cleaner and worth living.

- Smart Care: Providing up-to-date information of a patient to the doctor or caretaker help in giving the best care.

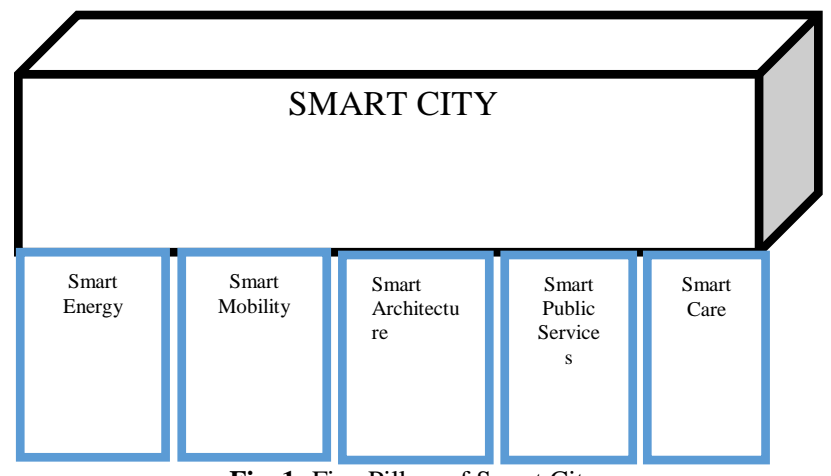

Fig. 1: Five Pillars of Smart City

\section{B. Smart Parking}

Living with smartness is the main agenda for today's life. To make your living a worth one, one need to ensure that the work he has been involved into must be done in an efficient manner. In this modern technological era everybody is being focused in inventing something new but for making the city smart, technology must be used, utilized and managed in proper way. Smart parking is one of the concern factors which have largely 
been ignored. With the increasing number of cars on the roads, lot of time gets consumed to park them as per the situation demand. Driving around after arriving at a mall, nobody wants to consume his half of the time gets wasted in searching for parking lot, it is sometimes frustrating. Parking lots are huge and multilayered, although for guidance smart parking pilots are there but they can send the drivers to the approximate location not to the definite parking space. IoT has come up with the best solution to get rid from such problems without human interventions.

\section{Smart Parking should carry the following traits}

- Whenever user demands for the nearest parking lots, the information should be provided to user.

- User must be informing about the empty lots in that parking area.

- No need to worry about the security of vehicle.

- Time is the biggest challenge that suffers a lot while looking for a parking space that has an adverse affect on cost, social interaction and the financial activities [8]. Human errors are biggest reason for causing accidents therefore many technologies came into power in order to avoid it. The objective of these technologies is to reduce the burden on the drivers, improve the traffic congestion and provide secure and reliable vehicle functions. The parking system which is based on token system is an inefficient, requires man power for its management and collecting parking payment [7]. However the management and enforcement system ensures the better effective and efficient method to deal with the security and meter reading. This works well for increasing revenue but requires human intervention which needs some capital [10]. The parking system which is currently in use is not efficient as it allow the drivers to park without any restrictions [9] which again not utilizing the parking facility. Through online parking reservation system, empty lots can be reserved by the driver and provide facilities like freedom of selecting payment modes to the drivers, which lessen the quantity of vehicles to be parked on the road and integrate the parking business.

\section{Smart City: Singapore}

Singapore was crowned as the smartest city on earth as per the Juniper Research (Basingstoke, UK). Singapore adopted many smart technologies to improve traffic problems for smart lighting, smartphone penetration and made various $\mathrm{Wi}-\mathrm{Fi}$ access points. Transport and energy are considered the main element in smart cities; the Juniper analysts evaluated the transportation section by subtracting the points for cities having large number of private vehicle from the public transportation analysis.

Singapore has taken the smart city to another level by the use of dynamic traffic lights, road sensors and smart parking. In the late 2014, the PM Lee Hsien Loong launched the program to attach sensors and cameras across the island to track everything from cleanliness to traffic. If people are smoking in an unauthorized space or throwing waste out of dustbins is detected by the sensors. Singapore earned highest marks for its smart mobility, policies, wireless connectivity and technology. Privacy and security are the main concern according to the Smart Nation Program therefore data can be anonymized when required. Singapore invested large amount on sensors and cameras to make the city smart, soon driverless cars will also introduced.

\section{Internet of Things (IoT): A Revolutionary Step}

Internet is the large medium to exchange information for communication. The device contribute in this interaction is not only a computer, tablet, mobile phones but a thing [1]. A thing can be any physical object present in the environment which can receive input and transform it into data. These data then forwarded to the server for further processing through internet.
Any object with the support of controller, sensor and actuators in the presence of internet is termed as Internet of Things [2]. Through IoT, it is possible to establish a global network communication by giving a unique identity to each object [3]. Here object can be a human or a thing.

\section{Proposed System}

\section{A. Smart Parking}

Living with smartness is the main agenda for today's life. To make living a worth one, one need to ensure that the work he has been involved into must be done in an efficient manner. In this modern technological era everybody is being focused in inventing something new but for making the city smart, technology must be used, utilized and managed in proper way.

Smart parking is one of the concern factors which have largely been ignored. With the increasing number of cars on the roads, lot of time gets consumed to park them as per the situation demand.

\section{B. Smart Parking using NB-IoT}

Narrow Band IoT Technology is one of the most innovative solutions among communication devices which operates under $3 \mathrm{GPP}$ and uses licensed bands to dealers. This technology provides greater network coverage by consuming less power and working efficiently even in the areas which is difficult to reach. It requires less power than Zigbee. Following is the comparison of NB-Iot and Zigbee under same environment[11]:

Table 1: Comparison of Energy Consumption

Table 1: Comparison of Energy Consumption
\begin{tabular}{|c|c|c|}
\hline Time & NB-IoT & Zigbee \\
\hline 1 month & $99 \%$ & $95 \%$ \\
\hline 5 months & $95 \%$ & $82 \%$ \\
\hline 9 months & $89 \%$ & $65 \%$ \\
\hline
\end{tabular}

With the advent of this technology, there is need to use additional gateways and allows large number of devices to connect with the network[13]. Also NB-IoT can be implemented at a low cost as it is directly applicable to Global System for Mobile communications (GSM) and the Universal Mobile Telecommunications System (UMTS) [5].

NB-IoT Smart parking solution gathers data like occupancy of parking lots,duration, etc and forward this to the service provider which in turn minimizes the economic losses and improves fee collection.

In 2017 Huawei along with China Unicom and TransInfo successfully launched a project of smart street parking based on NB-IoT where TransInfo provided parking management platforms and its related services, . network devices and chips were offered by Huawei and NB IoT modules by Quectel. China Unicom was responsible for providing E2E integration services. This allows the drivers to easily look for the parking spaces in real time and pay parking charges. Self-help payment system saves the manpower.

NB-IoT has been designed to achieve adjustable data rates with great multiplexing. NB-Iot works in two protocols: RRC_idle ie, in idle condition it saves the power and resources and another one is RRC_connected ie. Data intraction will be done directly[6].

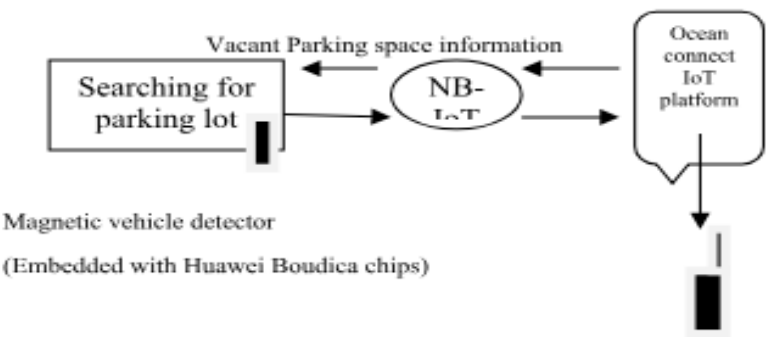

Parking Management System

Fig. 2: NB-IoT Enables Smart Parking 


\section{System based on Wireless Sensor}

Wireless Sensor Network (WSN) is easily installed. WSN based car parking system consist of cheap wireless sensors which is installed on each parking space collects the information regarding the parking status and direct it to the database. Acoustic, sound and light sensors are also used for the purpose of management. Magnetic and Ultrasound sensors are used for the reliable detection of empty lots [14]. Magnetometers with the modified min-max algorithm are used to detect the car whereas ultrasonic sensor algorithm is also utilized. However the magnetometers are environment sensitive so it can't be trusted in the unfavorable weather. Magnetometer is the technique which gives reading of Earth's magnetic field both in magnitude and direction affected by the vehicle. These sensors are only effective for ground parking, not suitable for the multilayered parking zones. Video sensors are very costly due to its large generation of data. The roadside parking system is managed by the small sensors which consumes less power [12] presents in each node respond with the changes in the magnetic field. The nodes get activated by the inter microcontroller to receive the parking status which saves power.

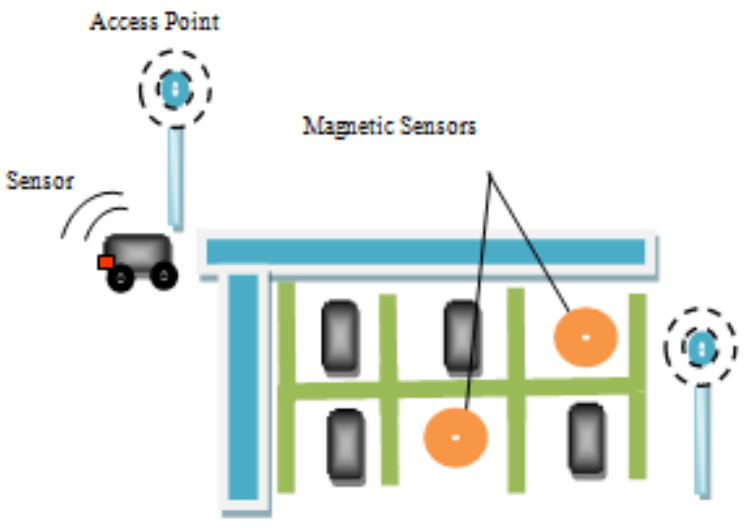

Fig. 3: Magnetic Sensor Operation

\section{System based on Wireless Sensor}

GPS involved system is used for allocating the nearest parking space. As it provide information about the current state so it cannot guarantee the same space would be available when the driver reaches. A solution regarding the management of past and present status is in [15]. For checking the availability of parking lots Poisson process is used. Through NAPA (Nearest Available Parking lot application) technique, it is possible to locate the parking destination but does not aware the user about the exact position of the vacant space.

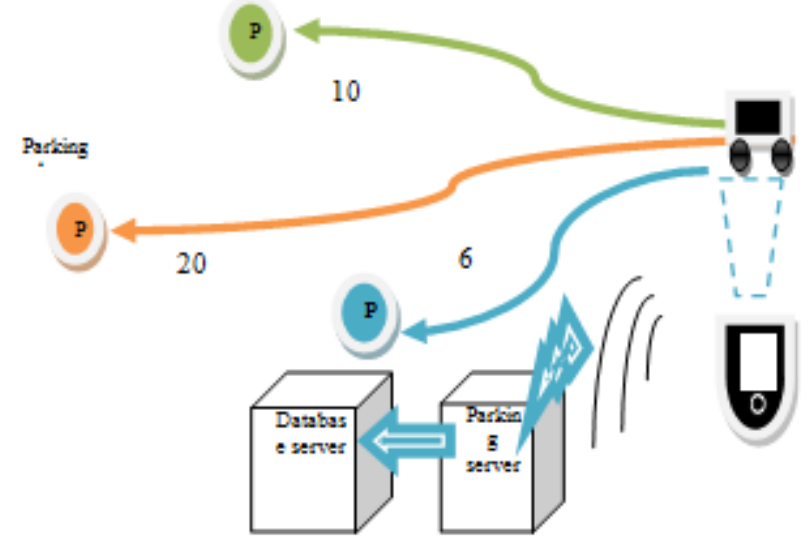

Fig. 4: NAPA Working Procedure

\section{E. A Smart Approach}

To fulfill the needy requirements, smart IOT techniques are used, as IOT has no limitations. User will get all the information about the locations of nearest parking lots from the GPS, so they can go for any suitable choice. Once they selected the area, all the information regarding it will be displayed on their smart devices. As we are talking about smartness and also going advance in our thoughts, advance booking parking system is also provided but has a limitation of 15 minutes i.e. the vehicle must be parked under 15 minutes if not the content will get reset.

a. The IR sensors are used for security purposes. If the IR rays found under the range of human body near that vehicle the message immediately delivered to the owner of the vehicle with the calculated distance. Once the driver parked the vehicle the sensors activate and give response to the server, the timer gets started. For the economic purpose if the vehicle overstays the limit, the owner have to pay the decided amount. So with the help of timer the message regarding it is sent to the owner 15 minutes before and on the completion of time. While exiting from the parking area user must reset the lot on the app, so that it can be allocated by others. For higher security Raspberry pi cameras can also be used in the entrance and the exit of the parking area.

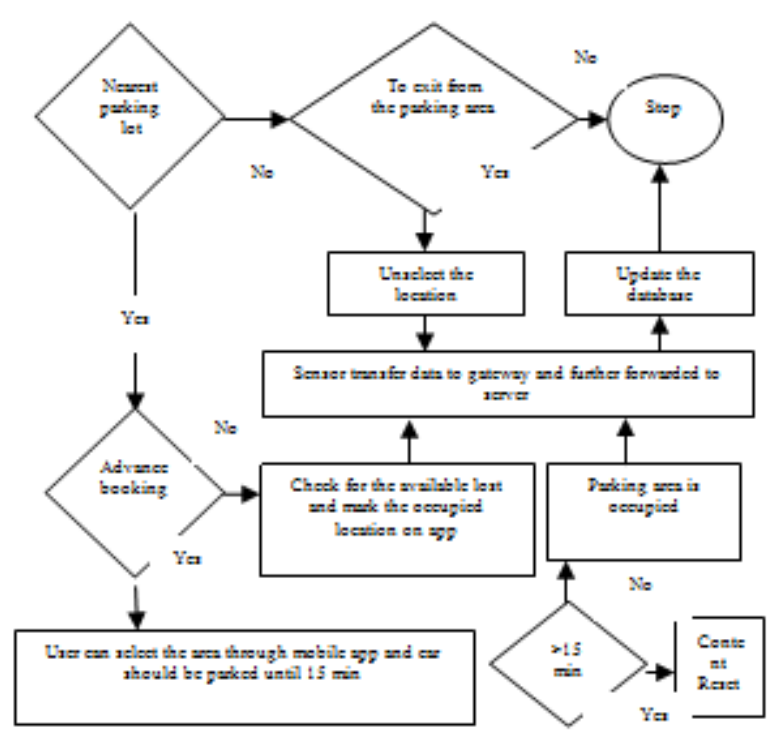

Fig. 5: Working procedure of smart parking approach

\section{Acknowledgment}

A big thanks to Mr Rahul Chauhan who helped us in writing this paper.

\section{References}

[1] Integrating Smart Objects and RFIDs into a Unified Internet of Things Architecture., Tselikas, N.D., Kosmatos, E.A and Boucouvalas, A.C. (2011)

[2] Designing the Internet of Things by Hakim Cassimally and Adrian McEwen.

[3] (2012) "Internet of Things" in RFID security context by Lal Das, $\mathrm{M}$ and Aggarwal.

[4] (Jayavardhana Gubbi, 2014), IEEE. “An Information Framework for Creating a Smart City Through Internet of Things".

[5] Wang J.Y., Wang X.Z., Wu Q. (2017). Core network service model and networking scheme oriented NB-IoT, Telecommunications Science, 4, pp. 149-151.

[6] https://ubidots.com/blog/nb-iot.

[7] (L. Bai, 2006), "A Smart Roadside Parking Navigation System Based On Sensor Networks For ITS" 
[8] "An agent-based platform for drivers and car parks negotiation", IEEE 2004 by (S.Y. Chou, 2004)

[9] "A Novel Parking Service Using Wireless Networks", IEEE by (M.C. Weigle, 2009)

[10] "An intelligent architecture for metropolitan area parking contro and toll collection", IEEE,(S.M. Mehmood, 2005).

[11] https://doi.org/10.3991/ijoe.v13i12.7886, Wei Zhai Xi'an Aeronautical University, Xi'an, China

[12] "Integrating Mobile Agent with Multi-Agent System for Intelligent Parking Negotiation and Guidance" by (L. Yang W, Integrating Mobile Agent with Multi-Agent System for Intelligent Parking Negotiation and Guidance, 2009.

[13] http://smartparkingsystem.com/en/nb-iot-sensors-applied-toparking-system.

[14] "Intelligent Parking Lot Application Using Wireless Sensor Networks", by (D. Yoon A. Ghosh, 2008) .

[15] "An Intelligent Secure And Privacy-Preserving Parking Scheme Through Vehicular Communications", IEEE by (L. Xiaodong, 2010).

[16] "Towards An Intelligent GPS-Based Vehicle Navigation System for Finding Street Parking Lots", IEEE,by (P.K. Atrey, 2007) 